\title{
Vacuum polarization of massive spinor fields in static black-string backgrounds
}

\author{
Owen Pavel Fernández Piedra* \\ Departamento de Física y Química, \\ Universidad de Cienfuegos, Carretera a Rodas, \\ Cuatro Caminos, s/n. Cienfuegos, Cuba. \\ Alejandro Cabo Montes de Ocat \\ Grupo de Física Teórica, ICIMAF, Calle E No. 309, \\ esq. a 15 Vedado, C. Habana,Cuba.
}

(Dated: November 3, 2018)

\begin{abstract}
The renormalized mean value of the quantum Lagrangian and the corresponding components of the Energy-Momentum tensor for massive spinor fields coupled to an arbitrary gravitational field configuration having cylindrical symmetry are analytically evaluated using the Schwinger-DeWitt approximation, up to second order in the inverse mass value. The general results are employed to explicitly derive compact analytical expressions for the quantum mean Lagrangian and EnergyMomentum tensor in the particular background of the Black-String space-time.
\end{abstract}

*Electronic address: opavel@ucf.edu.cu

${ }^{\dagger}$ Electronic address: cabo@icmf.inf.cu 


\section{INTRODUCTION}

In the absence of a full theory of quantum gravity, semiclasical gravity or Quantum Field theory in curved space-time is a well established physical theory that help us to know what are the expected behavior of gravitational system under the influence of the interaction between it and matter fields that obeys the laws of quantum theory. The research on this subject have received a great impulse since the Hawking discovery of black holes radiation [1].

One of the most important physical quantities to be determined in semiclassical gravity is the vacuum expectation value of the stress energy tensor $\left\langle T_{\mu}^{\nu}\right\rangle_{\text {ren }}$ of the quantum field . This quantity enters as a source in the semiclassical Einstein equations, which determines the changes in the gravitational background field due to its interaction with the quantum one (the backreaction). Up to know, there exist many works related to the calculation of the components of the renormalized stress tensor by different approaches. The main difficulty in the problem of calculate the components of $\left\langle T_{\mu}^{\nu}\right\rangle_{\text {ren }}$ is the dependence of this quantity on the metric tensor of the background gravitational field. For this reason it is impossible to have an exact analytical formula for this object. Except for some exact expressions for this quantity obtained for very special spacetimes, on which quantum matter fields propagates, and for boundary conditions with a high degree of symmetry [11, 12, 13, 14, 15], the majority of the techniques developed rest on approximate methods to build the energy momentum tensor or numerical computations of $\left\langle T_{\mu}^{\nu}\right\rangle_{\text {ren }}$ and the mean-squared field $\left\langle\varphi^{2}\right\rangle_{\text {ren }}$ [5, 16, 17, 18, 19, 20, 21]. One of the developed techniques is the Schwinger-De Witt expansion, that can be used to investigate effects like the vacuum polarization of massive fields in curved backgrounds. The Schwinger-DeWitt approximation results from an expansion of the effective action in powers of the inverse mass of the quantum field, and is valid to use it whenever the Compton's wavelenght of the field is less than the characteristic radius of curvature [6, 7, 8, 9, 22, 23].

In General Relativity, the formulation by Thorne of the hoop conjecture have as a consequence that cylindrical collapsing matter will not form a black hole. However, the hoop conjecture was given for spacetimes with a zero cosmological constant. In the presence of a negative cosmological constant one can expect the occurrence of major changes. Indeed, as was shown by Lemos and Zanchin [3] there are black hole solutions with cylindrical symmetry if a negative cosmological constant is present. Charged rotating black string solutions 
has many similarities with the Kerr-Newman black hole, apart from space-time being asymptotically anti-de Sitter in the radial direction (and not asymptotically flat). The existence of black strings suggests that they could be the final state of the collapse of matter having cylindrical symmetry.

The problems of determining $\left\langle\varphi^{2}\right\rangle_{\text {ren }}$ and investigate the renormalized stress tensor components for conformally coupled massless scalar fields in black String backgrounds were studied by DeBenedictis in [25, 26], who used the obtained $\left\langle T_{\mu}^{\nu}\right\rangle_{r e n}$ for the calculation of gravitational backreaction of the quantum field. In our previous papers [27, 28] we develop the Schwinger-DeWitt approximation for the stress energy tensor for a massive scalar field in the static cylindrical black hole background, showing that for a range of values of the coupling constant a violation of the weak energy condition can occur at the horizon of the considered space-time.

In this paper we consider the problem of evaluating the large mass limit of the renormalized vacuum expectation values of the Stress-Energy Tensor for a massive spinor field in a background space-time having cylindrical symmetry. The general results are applied to explicitly evaluate closed expression for those quantities in the special background formed by a neutral and non-rotating cylindrical Black String. In Section II first we collect some information about spinor field theory on a general curved background. In Section III we develop the Schwinger-DeWitt approximation for the one-loop effective action of the spinor field taking into account terms up to the second order in the inverse mass of the field and derive analytical expressions for the components of the renormalized vacuum expectation value of the stress-energy tensor of the quantum field. Section IV is devoted to review the metric tensor which solves the Einstein-Maxwell equations in the considered cylindric symmetry situation. Finally, employing the explicit form of the Black-String metric, closed expressions for the renormalized components of the Energy-Momentum tensor are derived in Section IV. These results can be used to study the vacuum polarization and the back-reaction of the quantum spinor field in the gravitational background. The last section contains some conclusive comments and future directions of this work.

In the following we use for the Riemann tensor, its contractions, and the covariant derivatives the sign conventions of Misner, Thorne and Wheeler [29]. Our units are such that $\hbar=c=G=1$. 


\section{FREE SPINOR FIELD IN CURVED SPACE}

Consider a single massive neutral spinor field in a gravitational background with metric tensor $g_{\mu \nu}$ in four dimensions. The action for the system is::

$$
S=S_{\text {gravity }}+S_{\text {matter }}
$$

with $S_{\text {gravity }}$ the Einstein-Hilbert action for the gravitational background field and $S_{\text {matter }}$ that of the Dirac field:

$$
S_{\text {gravity }}=\frac{1}{16 \pi} \int d^{4} x \sqrt{-g}(R-2 \Lambda)
$$

and:

$$
S_{\text {matter }}=\frac{i}{2} \int d^{4} x \sqrt{-g} \widetilde{\phi}\left[\gamma^{\mu} \nabla_{\mu} \phi+m \phi\right]
$$

where $m$ is the mass of the field, $\phi$ provides a spin representation of the vierbein group and $\widetilde{\phi}=\phi^{*} \gamma$, where ${ }^{*}$ means transpose. The Dirac matrices $\gamma$ and $\gamma^{\mu}$ satisfy the usual relation $\left[\gamma^{\mu}, \gamma^{\nu}\right]_{+}=2 g_{\mu \nu} \widehat{I}$, where $\widehat{I}$ is the $4 \times 4$ unit matrix. The covariant derivative of any spinor $\zeta$ obey the conmutation relations:

$$
\begin{gathered}
\nabla_{\mu} \nabla_{\nu} \zeta-\nabla_{\nu} \nabla_{\mu} \zeta=\frac{1}{2} \mathfrak{F}_{[\alpha, \beta]} R^{\alpha \beta}{ }_{\mu \nu} \\
\nabla_{\nu} \nabla_{\sigma} \nabla_{\mu} \zeta-\nabla_{\sigma} \nabla_{\nu} \nabla_{\mu} \zeta=\frac{1}{2} \mathfrak{F}_{[\alpha, \beta]} R_{\mu \sigma}^{\alpha \beta} \nabla_{\mu} \zeta+\nabla_{\rho} \zeta R_{\mu}{ }^{\rho}{ }_{\nu \sigma} \\
\nabla_{\sigma} \nabla_{\tau} \nabla_{\nu} \nabla_{\mu} \zeta-\nabla_{\tau} \nabla_{\sigma} \nabla_{\nu} \nabla_{\mu} \zeta=\frac{1}{2} \mathfrak{F}_{[\alpha, \beta]} R_{\sigma \tau}^{\alpha \beta} \nabla_{\nu} \nabla_{\mu} \zeta+\nabla_{\nu} \nabla_{\rho} \zeta R_{\mu}{ }^{\rho}{ }_{\sigma \tau}+\nabla_{\rho} \nabla_{\mu} \zeta R_{\nu}{ }^{\rho}{ }_{\sigma \tau}
\end{gathered}
$$

and so forth. In equations (4) to (6) :

$$
\mathfrak{F}_{[\alpha, \beta]}=\frac{1}{4}\left[\gamma_{\alpha}, \gamma_{\beta}\right]_{-}
$$

are the generators of the vierbein group, [ , ]_ is the commutator bracket, and :

$$
R_{\mu \nu}^{\alpha \beta}=h_{\sigma}^{\alpha} h^{\beta}{ }_{\tau} R_{\mu \nu}^{\sigma \tau}
$$

where $h_{\beta}^{\alpha}$ is the vierbein which satisfies $h_{\alpha \mu} h_{\nu}^{\alpha}=g_{\mu \nu}$. The covariant derivatives of $\gamma, \gamma^{\mu}$ and $\mathfrak{F}_{[\alpha, \beta]}$ vanishes.

By applying the functional derivative operation to the action (3) with respect to the spinor field $\phi$ we obtain the desired equation of motion for the field:

$$
\left(\gamma^{\mu} \nabla_{\mu}+m\right) \phi=0
$$




\section{SCHWINGER-DEWITT APPROXIMATION FOR THE RENORMALIZED EFFECTIVE ACTION OF THE SPINOR FIELD ON A GENERAL CURVED BACKGROUND}

In this section we give exact expressions for the renormalized effective lagrangian, the corresponding renormalized effective action, and the renormalized vaccum expectation value of the stress energy tensor for the neutral spinor field obeying equation (91) in the large mass limit. This approximation is known as the Schwinger-DeWitt one, and before applying this approach to the particular problem considered in this work we make the following remarks.

In the first place, we mention that the Schwinger-DeWitt technique is directly applicable to "minimal" second order differential operators ( acting on the super-field $\phi^{A}$ ) that have the general form:

$$
\hat{D}=\square-m^{2}+Q
$$

where $\square=g^{\mu \nu} \nabla_{\mu} \nabla_{\nu}$ is the covariant D'Alembert operator, $\nabla_{\mu}$ is the covariant derivative defined by means of some background conection $\mathfrak{C}_{\mu}(x)$,

$$
\nabla_{\mu} \phi^{A}=\partial_{\mu} \phi^{A}+\mathfrak{C}_{B \mu}^{A} \phi^{B}
$$

$g^{\mu \nu}$ is the metric of the background spacetime, $m$ is the mass parameter of the quantum field and $Q^{A}(x)$ is an arbitrary matrix playing the role of the potential.

The explicit form of the background affine connection $\mathfrak{C}_{B \mu}^{A}(x)$ that defines the covariant derivative (11) is not needed, only is necessary to know the commutator of covariant derivatives that defines curvature:

$$
\begin{gathered}
{\left[\nabla_{\alpha}, \nabla_{\beta}\right]_{-} \phi=\mathfrak{R}_{\alpha \beta} \phi} \\
\mathfrak{R}_{\alpha \beta}=\partial_{\alpha} \mathfrak{C}_{\beta}-\partial_{\beta} \mathfrak{C}_{\alpha}+\left[\mathfrak{C}_{\alpha}, \mathfrak{C}_{\beta}\right]_{-}
\end{gathered}
$$

In the spinor case of our interest the curvature has the form:

$$
\mathfrak{R}_{\alpha \beta}=\gamma^{\sigma} \gamma^{\tau} R_{\sigma \tau \alpha \beta}
$$

The usual formalism of Quantum Field Theory give an expression for the effective action of the quantum field $\phi$ as perturbation expansion in the number of loops:

$$
\Gamma(\Phi)=S(\Phi)+\sum_{k \geq 1} \Gamma_{(k)}(\Phi)
$$


where $S(\Phi)$ is the classical action of the free field. The one loop contribution of the field $\phi$ to the effective action is expressed in terms of the operator (10) as:

$$
\Gamma_{(1)}=-\frac{i}{2} \ln (s \operatorname{det} \hat{D})
$$

where $s \operatorname{det} \hat{F}=\exp (\operatorname{str} \ln \hat{F})$ is the functional Berezin superdeterminant [9] of the operator $\hat{F}$, and:

$$
\operatorname{str} \hat{F}=\left(-1^{i}\right) F_{i}^{i}=\int d^{4} x\left(-1^{A}\right) F_{A}^{A}(x)
$$

is the functional supertrace [9].

Using the Schwinger-DeWitt representation for the Greens function of the operator (10), we can obtain for the renormalized one loop effective action of the quantum field $\phi$ the expression:

$$
\Gamma_{(1) r e n}=\int d^{4} x \sqrt{-g} \mathfrak{L}_{r e n}
$$

where the renormalized effective Lagrangian reads:

$$
\mathfrak{L}_{\text {ren }}=\frac{1}{2(4 \pi)^{2}} \sum_{k=3}^{\infty} \frac{\operatorname{str} a_{k}(x, x)}{k(k-1)(k-2) m^{2(k-2)}}
$$

The coefficients $\left[a_{k}\right]=a_{k}\left(x, x^{\prime}\right)$, whose coincidence limit appears under the supertrace operation in (19) are the Hadamard-Minakshisundaram-DeWitt-Seeley coefficients (HMDS), whose complexity rapidly increases with $k$. As usual, the first three coefficients of the DeWitt-Schwinger expansion, $a_{0}, a_{1}$, and $a_{2}$, contribute to the divergent part of the action and can be absorbed in the classical gravitational action by renormalization of the bare gravitational and cosmological constants. Various authors have calculated some of the HDSM coefficients in exact form up to $k \leq 4[8,9,10]$.

Now the problem is that the differential operator $\hat{A}=\gamma^{\mu} \nabla_{\mu}+m$ that appears acting on the spinor field in (9) is not of the appropiate form (10). this problem is solved if one introduces a new spinor variable $\psi$ connected with $\phi$ by the relation $\phi=\gamma^{\sigma} \nabla_{\sigma} \psi-m \psi$ so that (9) take the form:

$$
\gamma^{\mu} \gamma^{\nu} \nabla_{\mu} \nabla_{\nu} \psi-{ }^{2} m \psi=0
$$

Using the properties of Dirac matrices and (14) we can establish the identity $\gamma^{\mu} \gamma^{\nu} \nabla_{\mu} \nabla_{\nu}=$ $\hat{I}\left(\square-\frac{1}{4} R\right)$ so that equation (9) takes the desired form:

$$
\left(\square-\frac{1}{4} R-m^{2}\right) \psi=0
$$


where the potential matrix can be easily identified as $Q=-\frac{1}{4} R \hat{I}$.

Restricting ourselves here to the terms proportional to $m^{-2}$, using integration by parts and the elementary properties of the Riemann tensor [9, 22], we obtain for the renormalized effective lagrangian ,

$$
\mathfrak{L}_{r e n}=\mathfrak{L}_{r e n}^{v a c}+\widetilde{\mathfrak{L}_{r e n}},
$$

where $\widetilde{\mathfrak{L}_{\text {ren }}}$ is a portion of the total lagrangian density that vanishes if the background spacetime is a vacuum solution of the classical Einstein equations and $\mathfrak{L}_{\text {ren }}^{v a c}$ is the remaining part of the total renormalized effective lagrangian density of the quantum field. The explicit expressions for this functions in the case of the spinor field considered in this work reads:

$$
\begin{aligned}
\widetilde{\mathfrak{L}_{r e n}} & =\frac{1}{192 \pi^{2} m^{2}}\left[\frac{1}{28} R_{\mu \nu} \square R^{\mu \nu}-\frac{3}{280} R \square R+\frac{1}{864} R^{3}-\frac{1}{180} R R_{\mu \nu} R^{\mu \nu}\right. \\
& -\frac{25}{756} R_{\nu}^{\mu} R_{\gamma}^{\nu} R_{\mu}^{\gamma}+\frac{47}{1260} R^{\mu \nu} R_{\gamma \varrho} R_{\mu \nu}^{\gamma \varrho}+\frac{19}{1260} R_{\mu \nu} R_{\sigma \gamma \varrho}^{\mu} R^{\nu \sigma \gamma \varrho} \\
& \left.-\frac{7}{1440} R R_{\mu \nu \gamma \varrho} R^{\mu \nu \gamma \varrho}\right],
\end{aligned}
$$

and

$$
\mathfrak{L}_{\text {ren }}^{\text {vac }}=\frac{1}{192 \pi^{2} m^{2}}\left[\frac{29}{7560} R_{\gamma \varrho}^{\mu \nu} R_{\mu \nu}^{\sigma \tau} R_{\sigma \tau}^{\gamma \varrho}-\frac{1}{108} R_{\mu \nu}^{\gamma \varrho} R_{\sigma \tau}^{\mu \nu} R_{\gamma}^{\sigma \tau} \varrho\right] .
$$

By standard functional differentiation of the effective action with respect to the metric, the renormalized Stress-Energy tensor is obtained according to the known formula:

$$
\left\langle T_{\mu \nu}\right\rangle_{r e n}=\frac{2}{\sqrt{-g}} \frac{\delta W_{r e n}}{\delta g^{\mu \nu}}
$$

The result can be written in a general form as

$$
\left\langle T_{\mu}{ }^{\nu}\right\rangle_{r e n}=V_{\mu}^{\nu}+D_{\mu}^{\nu}
$$

where again $D_{\mu}{ }^{\nu}$ is the part of the total stress tensor that vanishes if the background spacetime is a vacuum solution of the classical Einstein equations and $V_{\mu}{ }^{\nu}$ is the remaining 
part. The above tensors evaluated in this work take the forms

$$
\begin{aligned}
& D_{\mu}{ }^{\nu}=\frac{1}{96 \pi^{2} m^{2}}\left[-\frac{3}{280}\left(\nabla_{\mu} R \nabla^{\nu} R+\nabla^{\nu} \nabla_{\mu}(\square R)+\nabla_{\mu} \nabla^{\nu}(\square R)-2 \square^{2} R \delta_{\mu}{ }^{\nu}\right.\right. \\
& \left.-\frac{1}{2} \delta_{\mu}{ }^{\nu} \nabla_{\gamma} R \nabla^{\gamma} R-2 \square R \nabla^{\nu} \nabla_{\mu} R\right)+\frac{1}{28}\left[\nabla_{\mu} R_{\gamma \lambda} \nabla^{\nu} R^{\gamma \lambda}-\nabla^{\nu} R_{\gamma \lambda} \nabla^{\lambda} R_{\mu}{ }^{\gamma}\right. \\
& -\nabla_{\mu} R_{\gamma \lambda} \nabla^{\lambda} R^{\gamma \nu}+\nabla^{\gamma} R_{\gamma \lambda} \nabla^{\nu} R_{\mu}{ }^{\lambda}+\nabla^{\gamma} R_{\gamma \lambda} \nabla_{\mu} R^{\lambda \nu}+\nabla^{\gamma} \nabla^{\nu}\left(\square R_{\gamma \mu}\right)-\square^{2} R_{\mu}{ }^{\nu} \\
& +\nabla^{\gamma} \nabla_{\mu}\left(\square R_{\gamma}^{\nu}\right)-\frac{1}{2} \nabla_{\varrho} R_{\gamma \lambda} \nabla^{\varrho} R^{\gamma \lambda} \delta_{\mu}^{\nu}-\nabla^{\gamma} \nabla^{\lambda}\left(\square R_{\gamma \lambda}\right) \delta_{\mu}{ }^{\nu}+\nabla_{\lambda} \nabla^{\nu} R_{\gamma \mu} R^{\gamma \lambda} \\
& +\nabla_{\lambda} \nabla_{\mu} R_{\gamma}{ }^{\nu} R^{\gamma \lambda}-\left(-\nabla_{\lambda} \nabla_{\sigma} R_{\gamma}^{\lambda}{ }^{\sigma \nu} R_{\mu}{ }^{\gamma}+\frac{1}{2} \nabla^{\nu} \nabla_{\gamma} R-R^{\lambda \sigma} R_{\gamma}{ }^{\nu} \lambda \sigma\right) R_{\mu}{ }^{\gamma} \\
& \text { - } \left.\nabla^{\gamma} \nabla^{\nu} R_{\gamma \lambda} R_{\mu}^{\gamma}+R_{\gamma \lambda} R^{\gamma \lambda} R_{\mu}^{\gamma}-\square R_{\gamma \mu} R^{\gamma \nu}-\nabla^{\lambda} \nabla_{\mu} R_{\gamma \lambda} R^{\gamma \nu}\right] \\
& -\frac{25}{756}\left[\frac{3}{2} \nabla^{\nu} R_{\gamma \lambda} \nabla^{\lambda} R_{\mu}{ }^{\gamma}-\frac{3}{2} \nabla^{\gamma} R_{\gamma \lambda} \nabla^{\varrho} R_{\varrho}{ }^{\lambda} \delta_{\mu}{ }^{\nu}-\frac{3}{2} \nabla_{\varrho} R_{\gamma \lambda} \nabla^{\lambda} R^{\gamma \varrho} \delta_{\mu}{ }^{\nu}+\frac{3}{2} \nabla_{\lambda} \nabla^{\nu} R_{\gamma \mu} R^{\gamma \lambda}\right. \\
& +\frac{3}{2} \nabla_{\lambda} \nabla_{\mu} R_{\gamma}{ }^{\nu} R^{\gamma \lambda}-\frac{3}{2} \nabla^{\lambda} \nabla_{\varrho} R_{\gamma \lambda} R^{\gamma \varrho} \delta_{\mu}{ }^{\nu}+\frac{3}{2} \nabla^{\lambda} \nabla^{\nu} R_{\gamma \lambda} R_{\mu}{ }^{\gamma}-\frac{3}{2}\left(\nabla_{\lambda} \nabla_{\sigma} R_{\gamma}^{\lambda}{ }^{\sigma \nu} R_{\mu}{ }^{\gamma}\right. \\
& \left.+\frac{1}{2} \nabla^{\nu} \nabla_{\gamma} R-R^{\lambda \sigma} R_{\gamma \lambda \sigma}^{\nu}+R_{\gamma \lambda} R^{\gamma \lambda}\right) R_{\mu}{ }^{\gamma}+\frac{3}{2} \nabla^{\gamma} R_{\gamma \lambda} \nabla_{\mu} R^{\lambda \nu} \\
& +\frac{3}{2} \nabla^{\lambda} \nabla_{\mu} R_{\gamma \lambda} R^{\gamma \nu}-\frac{3}{2} \square R_{\gamma \mu} R^{\gamma \nu}-\frac{3}{2} \nabla_{\varrho} \nabla^{\gamma} R_{\gamma \lambda} R^{\lambda \varrho} \delta_{\mu}{ }^{\nu}+R_{\gamma \lambda} R_{\varrho}^{\gamma} R^{\lambda \varrho} \delta_{\mu}{ }^{\nu} \\
& \left.-3 R_{\gamma \lambda} R_{\mu}{ }^{\gamma} R^{\lambda \nu}+\frac{3}{2} \nabla_{\mu} R_{\gamma \lambda} \nabla^{\lambda} R^{\gamma \nu}-3 \nabla_{\gamma} R_{\gamma \mu} \nabla^{\lambda} R^{\gamma \nu}+\frac{3}{2} \nabla^{\gamma} R_{\gamma \lambda} \nabla^{\nu} R_{\mu}{ }^{\lambda}\right] \\
& +\frac{47}{1260}\left(\nabla^{\gamma} R_{\gamma \mu} \nabla_{\lambda} R_{\lambda}{ }^{\nu}+\nabla_{\lambda} R_{\gamma}{ }^{\nu} \nabla^{\gamma} R_{\mu}{ }^{\lambda}-2 \nabla^{\gamma} R_{\gamma \lambda} \nabla^{\lambda} R_{\mu}{ }^{\nu}-\nabla^{\nu} R_{\gamma \lambda} \nabla^{\varrho} R_{\varrho}{ }^{\gamma \lambda}{ }_{\mu}\right. \\
& +\nabla_{\varrho} R_{\gamma \lambda} \nabla_{\mu} R^{\gamma \varrho \lambda \nu}+2 \nabla_{\varrho} R_{\gamma \lambda} \nabla^{\sigma} R_{\sigma}^{\gamma \lambda \varrho} \delta_{\mu}{ }^{\nu}-\nabla_{\lambda} \nabla_{\gamma} R_{\mu}{ }^{\nu} R^{\gamma \lambda}+\nabla^{\varrho} \nabla^{\nu} R_{\gamma \lambda \varrho \mu} R^{\gamma \lambda} \\
& -\square R_{\gamma \mu \lambda}{ }^{\nu} R^{\gamma \lambda}+\nabla^{\varrho} \nabla_{\mu} R_{\gamma}{ }^{\nu}{ }_{\lambda \varrho} R^{\gamma \lambda}-\nabla^{\lambda} \nabla^{\sigma} R_{\gamma \lambda \varrho \sigma} R^{\gamma \varrho} \delta_{\mu}{ }^{\nu}+\frac{1}{2} \nabla^{\gamma} \nabla_{\lambda} R_{\gamma}{ }^{\nu} R_{\mu}{ }^{\lambda} \\
& +\frac{1}{2} \nabla_{\lambda} \nabla^{\gamma} R_{\gamma}{ }^{\nu} R_{\mu}{ }^{\lambda}+\frac{1}{2} \nabla^{\gamma} \nabla_{\lambda} R_{\gamma \mu} R^{\lambda \nu}+\frac{1}{2} \nabla_{\lambda} \nabla^{\gamma} R_{\gamma \mu} R^{\lambda \nu}+\frac{1}{2} R_{\gamma \lambda} R_{\varrho \sigma} R^{\gamma \varrho \lambda \sigma} \delta_{\mu}{ }^{\nu} \\
& -\frac{3}{2} R_{\gamma \lambda} R_{\varrho}{ }^{\nu} R_{\mu}^{\gamma \varrho \lambda}-\frac{3}{2} R_{\gamma \lambda} R_{\varrho \mu} R^{\gamma \varrho \lambda \nu}-\nabla^{\gamma} \nabla^{\lambda} R_{\gamma \lambda} R_{\mu}{ }^{\nu}+\nabla_{\varrho} \nabla^{\nu} R_{\gamma \lambda} R_{\mu}^{\gamma \varrho \lambda} \\
& +\nabla_{\varrho} \nabla_{\mu} R_{\gamma \lambda} R^{\gamma \varrho \lambda \nu}-\nabla_{\sigma} \nabla_{\varrho} R_{\gamma \lambda} R^{\gamma \sigma \lambda \varrho} \delta_{\mu}{ }^{\nu}-\square R_{\gamma \lambda} R_{\mu}^{\gamma}{ }^{\lambda \nu}-\nabla_{\mu} R_{\gamma \lambda} \nabla^{\varrho} R_{\varrho}^{\gamma \lambda \nu} \\
& \left.+\nabla_{\varrho} R_{\gamma \lambda} \nabla^{\nu} R_{\mu}{ }^{\gamma \varrho \lambda}-2 \nabla_{\varrho} R_{\gamma \lambda} \nabla^{\varrho} R_{\mu}^{\gamma}{ }^{\lambda \nu}\right)+\frac{19}{1260}\left(2 \nabla_{\lambda} R_{\gamma \mu} \nabla^{\varrho} R_{\varrho}{ }^{\nu \gamma \lambda}-2 \nabla^{\gamma} R_{\gamma \lambda} \nabla^{\varrho} R_{\varrho}{ }^{\nu \lambda} \mu\right. \\
& -2 \nabla_{\varrho} R_{\gamma \lambda} \nabla^{\lambda} R_{\mu}^{\gamma}{ }^{\varrho \nu}-2 \nabla_{\varrho} \nabla^{\gamma} R_{\gamma \lambda \mu}{ }^{\nu} R^{\lambda \varrho}-2 \nabla^{\gamma} \nabla^{\varrho} R_{\gamma \lambda \varrho}{ }^{\nu} R_{\mu}{ }^{\lambda}+2 \nabla_{\varrho} \nabla_{\lambda} R_{\gamma \mu} R^{\gamma \varrho \lambda \nu} \\
& \left.+R_{\gamma \mu} R_{\lambda \varrho \sigma}{ }^{\nu} R^{\gamma \sigma \lambda \varrho}-2 \nabla_{\lambda} R_{\gamma \mu} \nabla^{\varrho} R_{\varrho}^{\gamma \lambda \nu}-2 \nabla^{\gamma} \nabla_{\varrho} R_{\gamma \lambda} R_{\mu}^{\lambda}{ }^{\varrho \nu}\right) \\
& -\frac{1}{180}\left(\nabla^{\nu} R \nabla^{\gamma} R_{\gamma \mu}+\nabla_{\mu} R \nabla^{\gamma} R_{\gamma}^{\nu}+2 \nabla^{\nu} R_{\gamma \lambda} \nabla_{\mu} R^{\gamma \lambda}-\square R R_{\mu}{ }^{\nu}\right. \\
& +\nabla_{\gamma} R \nabla^{\nu} R_{\mu}{ }^{\gamma}+\nabla_{\gamma} R \nabla_{\mu} R^{\gamma \nu}-2 \nabla_{\gamma} R \nabla^{\gamma} R_{\mu}{ }^{\nu}+R \nabla^{\gamma} \nabla^{\nu} R_{\gamma \mu}+R \nabla^{\gamma} \nabla_{\mu} R_{\gamma}{ }^{\nu}
\end{aligned}
$$




$$
\begin{aligned}
& -R \nabla_{\lambda} \nabla_{\gamma} R_{\mu}^{\lambda}{ }^{\gamma \nu}-\frac{1}{2} R \nabla^{\nu} \nabla_{\mu} R+R R^{\lambda \gamma} R_{\mu \lambda}{ }_{\gamma}^{\nu}-R R_{\mu \lambda} R^{\lambda \nu}-2 \nabla_{\gamma} R \nabla^{\lambda} R_{\lambda}^{\gamma} \delta_{\mu}{ }^{\nu} \\
& +\nabla^{\nu} \nabla_{\mu} R_{\gamma \lambda} R^{\gamma \lambda}+\nabla_{\mu} \nabla^{\nu} R_{\gamma \lambda} R^{\gamma \lambda}+\nabla_{\lambda} \nabla_{\gamma} R R^{\gamma \lambda} \delta_{\mu}{ }^{\nu}-2 \square R_{\gamma \lambda} R^{\gamma \lambda} \delta_{\mu}{ }^{\nu} \\
& \left.+\frac{1}{2} R R_{\gamma \lambda} R^{\gamma \lambda} \delta_{\mu}{ }^{\nu}+\nabla^{\nu} \nabla_{\gamma} R R_{\mu}{ }^{\gamma}-2 R R_{\gamma}{ }^{\nu} R_{\mu}{ }^{\gamma}+\nabla_{\mu} \nabla_{\gamma} R R^{\gamma \nu}-R_{\gamma \lambda} R^{\gamma \lambda} R_{\mu}{ }^{\nu}\right) \\
& -\frac{7}{1440}\left(+4 \nabla_{\gamma} R \nabla^{\lambda} R_{\lambda \mu}{ }^{\gamma \nu}+4 \nabla_{\gamma} R \nabla^{\lambda} R_{\lambda}^{\nu \gamma}{ }_{\mu}+2 R \nabla^{\gamma} \nabla^{\lambda} R_{\gamma \mu \lambda}{ }^{\nu}+2 R \nabla^{\gamma} \nabla^{\lambda} R_{\gamma}{ }^{\nu} \lambda \mu\right. \\
& -\frac{1}{2} R R_{\gamma \lambda \varrho \sigma} R^{\gamma \lambda \varrho \sigma} \delta_{\mu}^{\nu}+R_{\mu}{ }^{\nu} R_{\gamma \lambda \varrho \sigma} R^{\gamma \lambda \varrho \sigma}+2 R R_{\gamma \lambda \varrho}{ }^{\nu} R_{\mu}^{\gamma \lambda \varrho}+2 \nabla_{\lambda} \nabla_{\gamma} R R_{\mu}^{\gamma}{ }^{\lambda \nu} \\
& \left.+2 \nabla_{\lambda} \nabla_{\gamma} R R_{\mu}^{\gamma \nu \lambda}-2 \nabla_{\varrho} R_{\gamma \lambda} \nabla^{\varrho} R^{\gamma \lambda} \delta_{\mu}{ }^{\nu}-R \nabla^{\gamma} \nabla^{\lambda} R_{\gamma \lambda} \delta_{\mu}{ }^{\nu}\right) \\
& -\frac{1}{144}\left(\nabla_{\mu} R \nabla^{\nu} R+R \nabla^{\nu} \nabla_{\mu} R+\frac{1}{12} R^{3} \delta_{\mu}{ }^{\nu}-R \square R \delta_{\mu}{ }^{\nu}-\frac{1}{2} R^{2} R_{\mu}{ }^{\nu}-\nabla_{\gamma} R \nabla^{\gamma} R \delta_{\mu}{ }^{\nu}\right)
\end{aligned}
$$

and:

$$
\begin{aligned}
& V_{\mu}{ }^{\nu}=\frac{1}{96 \pi^{2} m^{2}}\left[-\frac{7}{1440}\left(2 \square R_{\gamma \lambda \varrho \sigma} R^{\gamma \lambda \varrho \sigma} \delta_{\mu}{ }^{\nu}+2 \nabla_{\tau} R_{\gamma \lambda \varrho \sigma} \nabla^{\tau} R^{\gamma \lambda \varrho \sigma} \delta_{\mu}{ }^{\nu}-2 \nabla^{\nu} R_{\gamma \lambda \varrho \sigma} \nabla_{\mu} R^{\gamma \lambda \varrho \sigma}\right.\right. \\
& \left.-\nabla^{\nu} \nabla_{\mu} R_{\gamma \lambda \varrho \sigma} R^{\gamma \lambda \varrho \sigma}-\nabla_{\mu} \nabla^{\nu} R_{\gamma \lambda \varrho \sigma} R^{\gamma \lambda \varrho \sigma}\right)+\frac{87}{7560}\left(-2 \nabla^{\varrho} R_{\gamma \lambda \varrho}{ }^{\nu} \nabla^{\sigma} R_{\sigma \mu}^{\gamma \lambda}\right. \\
& -\nabla^{\varrho} \nabla_{\sigma} R_{\gamma \lambda \varrho}^{\nu} R_{\mu}^{\gamma \lambda \sigma}-\nabla^{\varrho} \nabla_{\sigma} R_{\gamma \lambda \varrho \mu} R^{\gamma \lambda \sigma \nu}-\nabla_{\sigma} \nabla^{\gamma} R_{\gamma}^{\nu}{ }_{\lambda \varrho} R_{\mu}^{\lambda \varrho \sigma}-R_{\gamma \lambda \varrho \mu} R_{\sigma \tau}^{\varrho \nu} R^{\gamma \lambda \sigma \tau} \\
& \left.-\nabla_{\sigma} \nabla^{\gamma} R_{\gamma \mu \lambda \varrho} R^{\lambda \varrho \sigma \nu}+\frac{1}{6} R_{\gamma \lambda \varrho \sigma} R_{\tau \chi}^{\gamma \lambda} R^{\varrho \sigma \tau \chi} \delta_{\mu}^{\nu}-2 \nabla_{\sigma} R_{\gamma \lambda \varrho}{ }^{\nu} \nabla^{\varrho} R_{\mu}^{\gamma \lambda \sigma}\right) \\
& +\frac{19}{1260}\left(-\frac{1}{2} \square R_{\gamma \mu \lambda \varrho} R^{\gamma \nu \lambda \varrho}+\nabla^{\nu} R_{\gamma \lambda \varrho \sigma} \nabla^{\sigma} R_{\mu}^{\gamma \lambda \varrho}-\nabla^{\gamma} R_{\gamma \lambda \varrho \sigma} \nabla^{\nu} R_{\mu}^{\lambda}{ }^{\varrho \sigma}\right. \\
& -\nabla_{\sigma} R_{\gamma \lambda \varrho \mu} \nabla^{\sigma} R^{\gamma \lambda \varrho \nu}-\frac{1}{2} \nabla^{\gamma} R_{\gamma \lambda \varrho \sigma} \nabla^{\tau} R_{\tau}^{\lambda \varrho \sigma} \delta_{\mu}{ }^{\nu}+\nabla_{\sigma} \nabla^{\nu} R_{\gamma \mu \lambda \varrho} R^{\gamma \sigma \lambda \varrho} \\
& -\frac{1}{2} \nabla^{\lambda} \nabla_{\tau} R_{\gamma \lambda \varrho \sigma} R^{\gamma \tau \varrho \sigma} \delta_{\mu}{ }^{\nu}-\frac{1}{2} \nabla_{\tau} R_{\gamma \lambda \varrho \sigma} \nabla^{\sigma} R^{\gamma \lambda \varrho \tau} \delta_{\mu}{ }^{\nu}+\frac{1}{2} \nabla_{\tau} \nabla^{\gamma} R_{\gamma \lambda \varrho \sigma} R^{\lambda \tau \varrho \sigma} \delta_{\mu}{ }^{\nu} \\
& \left.+\nabla_{\lambda} \nabla^{\nu} R_{\gamma \lambda \varrho \sigma} R_{\mu}^{\gamma}{ }^{\varrho \sigma}-\frac{1}{2} \square R_{\gamma}^{\nu}{ }^{\nu} R^{\gamma}{ }_{\mu}{ }^{\lambda \varrho}\right)-\frac{1}{108}\left(3 \nabla^{\gamma} R_{\gamma \lambda \varrho \mu} \nabla^{\sigma} R_{\sigma}^{\varrho \lambda \nu}\right. \\
& +\frac{1}{2} R_{\gamma \lambda \varrho \sigma} R_{\tau}^{\gamma} \chi^{\varrho} R^{\lambda \tau \sigma \alpha} \delta_{\mu}{ }^{\nu}+3 \nabla^{\gamma} R_{\gamma \lambda \varrho \sigma} \nabla^{\sigma} R^{\gamma \nu \varrho}-\frac{3}{2} \nabla_{\sigma} \nabla_{\varrho} R_{\gamma \mu \lambda}{ }^{\nu} R^{\gamma \sigma \lambda \varrho} \\
& +\frac{3}{2} \nabla_{\sigma} \nabla^{\varrho} R_{\gamma \lambda \varrho}^{\nu} R_{\mu}^{\gamma \sigma \lambda}+\frac{3}{2} \nabla_{\sigma} \nabla^{\varrho} R_{\gamma \mu \lambda \varrho} R^{\gamma \sigma \lambda \nu}-3 R_{\gamma \lambda \varrho \mu} R_{\sigma \tau}^{\lambda \nu} R^{\gamma \sigma \varrho \tau} \\
& +\frac{3}{2} \nabla_{\sigma} \nabla^{\lambda} R_{\gamma \lambda \varrho}{ }^{\nu} R_{\mu}^{\gamma}{ }^{\varrho \sigma}-\frac{3}{2} \nabla^{\lambda} \nabla^{\sigma} R_{\gamma \lambda \varrho \sigma} R_{\mu}^{\gamma}{ }^{\varrho \nu}+\frac{3}{2} \nabla^{\lambda} \nabla_{\sigma} R_{\gamma \lambda \varrho \mu} R^{\gamma \nu \varrho \sigma} \\
& \left.+3 \nabla^{\gamma} R_{\gamma \lambda \varrho \sigma} \nabla^{\sigma} R_{\mu}^{\lambda}{ }^{\varrho \nu}-\frac{3}{2} \nabla^{\lambda} \nabla^{\sigma} R_{\gamma \lambda \varrho \sigma} R_{\mu}^{\gamma \nu \varrho}+3 \nabla_{\sigma} R_{\gamma \lambda \varrho}{ }^{\nu} \nabla^{\lambda} R_{\mu}^{\gamma}{ }^{\varrho \sigma}\right] \\
& \left.-\frac{3}{2} \nabla_{\sigma} \nabla_{\varrho} R_{\gamma \lambda \mu}^{\nu} R^{\gamma \sigma \lambda \varrho}\right)
\end{aligned}
$$


We should stress that a similar calculation was first performed by Matyjasek for ReissnerNordstrom space-time in reference [22] and for a general space-time in [23]. Our results are somewhat different from that of Matyjasek, but in view of the existence of many tensor identities relating the metric tensor, the Riemann tensor, its contractions and its covariant derivatives, we expect the two results will be equivalent. The formulas obtained in reference [23] and those obtained by us, when applied to Schwarshild, Reissner-Nordstrom and Kerr space-time shows identical results for the components of the renormalized stress tensor. However [24], in the case of the black string space-time, as we will show later in this paper, there are some minor differences in some numerical coefficients in the results for the massive scalar and spinor fields when compared with that obtained employing the formulas obtained by Matyjasek in reference [22]. The origin of this minor differences remain unclear for us at the moment of writing this report.

\section{THE CYLINDRICAL BLACK HOLE}

The charged rotating Black String or cylindrical black hole spacetime is an stationary cylindrically symmetric solution of the Einstein-Maxwell equations derived from the action (see Ref. [3] ):

$$
S=S_{\text {gravity }}+S_{\text {em }}
$$

where $S_{\text {gravity }}$ is given by (2) and:

$$
S_{e m}=-\frac{1}{16 \pi} \int d^{4} x \sqrt{-g} F^{\mu \nu} F_{\mu \nu}
$$

correspond to the presence of an electromagnetic field described by the Maxwell tensor:

$$
F_{\mu \nu}=\partial_{\mu} A_{\nu}-\partial_{\nu} A_{\mu}
$$

$A_{\mu}$ being the vector potential. The metric element in a cylindrical coordinate system $\left(x^{0}, x^{1}, x^{2}, x^{3}\right)=(t, \rho, \varphi, z)$ with $-\infty<t<\infty, 0 \leq \rho<\infty,-\infty<z<\infty, 0 \leq \varphi<2 \pi$ adequate for the geometry of interest results.

$$
\begin{aligned}
d s^{2}= & -\left(\alpha^{2} \rho^{2}-\frac{2(M+\Omega)}{\alpha \rho}+\frac{4 Q^{2}}{\alpha^{2} \rho^{2}}\right) d t^{2}-\frac{16 J}{3 \alpha \rho}\left(1-\frac{2 Q^{2}}{(M+\Omega) \alpha \rho}\right) d t d \varphi \\
& +\left[\rho^{2}+\frac{4(M-\Omega)}{\alpha^{3} \rho}\left(1-\frac{2 Q^{2}}{(M+\Omega) \alpha \rho}\right)\right] d \varphi^{2} \\
& +\frac{1}{\alpha^{2} \rho^{2}-\frac{2(3 \Omega-M)}{\alpha \rho}+\frac{4 Q^{2}(3 \Omega-M)}{\alpha^{2} \rho^{2}(\Omega+M)}} d \rho^{2}+\alpha^{2} \rho^{2} d z^{2}
\end{aligned}
$$


where $M, Q$, and $J$ are the mass, charge, and angular momentum per unit length of the string respectively. $\Omega$ is given by

$$
\Omega=\sqrt{M^{2}-\frac{8 J^{2} \alpha^{2}}{9}}
$$

The constant $\alpha$ is defined as follows:

$$
\alpha^{2}=-\frac{1}{3} \Lambda
$$

where $\Lambda$ is a negative Cosmological Constant. The corresponding metric element for the static spacetime follow form to relation (31):

$$
d s^{2}=-\left(\alpha^{2} \rho^{2}-\frac{4 M}{\alpha \rho}\right) d t^{2}+\frac{1}{\left(\alpha^{2} \rho^{2}-\frac{4 M}{\alpha \rho}\right)} d \rho^{2}+\rho^{2} d \varphi^{2}+\alpha^{2} \rho^{2} d z^{2} .
$$

As we can see from (34), the considered metric has an event horizon located at $\rho_{H}=\frac{\sqrt[3]{4 M}}{\alpha}$. The apparent singular behavior at this horizon is a coordinate effect and not a true one. The only true singularity is a polynomial one at the origin, as can it be seen after calculating the Kretschmann scalar, that results in

$$
K=R_{\alpha \beta \xi \gamma} R^{\alpha \beta \xi \gamma}=24 \alpha^{4}\left(1+\frac{M^{2}}{\alpha^{6} \rho^{6}}\right) .
$$

\section{RENORMALIZED STRESS-ENERGY TENSOR FOR SPINOR FIELDS IN A BLACK STRING BACKGROUND}

In the space-time of a static Black String metric given by (34) simple results were obtained for the renormalized Stress Tensor of massive spinor field coupled to the background gravitational field. After a direct calculation, we evaluate for the total stress tensor:

$$
\begin{gathered}
T_{t}{ }^{t}=\frac{1}{40320 \pi^{2} m^{2} \alpha^{3} \rho^{9}}\left(719 \alpha^{9} \rho^{9}-2976 \alpha^{3} M^{2} \rho^{3}+19072 M^{3}\right) \\
T_{z}^{z}=T_{\varphi}^{\varphi}=\frac{1}{40320 \pi^{2} m^{2} \alpha^{3} \rho^{9}}\left(719 \alpha^{9} \rho^{9}-3552 \alpha^{3} M^{2} \rho^{3}+28288 M^{3}\right), \\
T_{\rho}{ }^{\rho}=\frac{1}{40320 \pi^{2} m^{2} \alpha^{3} \rho^{9}}\left(719 \alpha^{9} \rho^{9}+3360 \alpha^{3} M^{2} \rho^{3}-6272 M^{3}\right) .
\end{gathered}
$$

It is interesting to evaluate the above components of the stress tensor at the event horizon of the black string. We obtain the following very simple results:

$$
T_{t}{ }^{t}{ }_{\text {horizon }}=\left.T_{\rho}{ }^{\rho}\right|_{\text {horizon }}=2.06 \cdot 10^{-2} \frac{\alpha^{6}}{\pi^{2} m^{2}}
$$




$$
\left.T_{z}{ }^{z}\right|_{\text {horizon }}=\left.T_{\varphi}^{\varphi}\right|_{\text {horizon }}=42.9 \frac{\alpha^{6}}{\pi^{2} m^{2}}
$$

At this point is useful to make an analysis of the violations or not of any of the well defined Energy Conditions that it is expected to be satisfied for any classical form of matter. We recall in the following the statements of the main Energy Conditions as appear in the literature [30]:

Weak energy Condition: The weak energy condition states that the energy density of any matter distribution, as measured by any observer in spacetime, must be nonnegative. Because an observer with four velocity $V^{\alpha}$ measures the energy density to be $T_{\alpha \beta} V^{\alpha} V^{\beta}$, we must have:

$$
T_{\alpha \beta} V^{\alpha} V^{\beta} \geq 0
$$

for any future-directed timelike vector $V^{\alpha}$. This condition implies (in terms of energy density $\varepsilon$ and principal pressures $p_{i}$ ) that:

$$
\varepsilon \geq 0 \quad \text { and } \quad \forall i, \quad \varepsilon+p_{i} \geq 0
$$

Null energy Condition: The null energy condition makes the same statement as the weak form, except that $V^{\alpha}$ is replaced by an arbitrary, future-directed null vector $k^{\alpha}$. Thus:

$$
T_{\alpha \beta} k^{\alpha} k^{\beta} \geq 0
$$

is the statement of the null energy condition. This condition implies:

$$
\forall i, \quad \varepsilon+p_{i} \geq 0
$$

Strong energy Condition: The statement of the Strong Energy Condition is:

$$
\left(T_{\alpha \beta}-\frac{1}{2} T g_{\alpha \beta}\right) V^{\alpha} V^{\beta} \geq 0
$$

where $V^{\alpha}$ is any future-directed, normalized, timelike vector $V^{\alpha}$. The strong energy condition therefore implies:

$$
\forall i, \quad \varepsilon+p_{i} \geq 0 \quad \text { and } \quad \varepsilon+\sum_{i} p_{i} \geq 0
$$

By virtue of the Einstein field equations, the strong energy condition is really a statement about the Ricci tensor. 
Dominant energy Condition: The dominant energy condition embodies the notion that matter should flow along timelike or null world lines. Its precise statement is that if $V^{\alpha}$ is an arbitrary, future-directed, timelike vector field, then:

$$
T_{\alpha \beta} V^{\alpha} V^{\beta} \geq 0 \quad \text { and } \quad-T_{\beta}^{\alpha} V^{\beta} \quad \text { is not spacelike }
$$

for any future-directed timelike vector $V^{\alpha}$. This condition implies (in terms of energy density $\varepsilon$ and principal pressures $p_{i}$ ) that:

$$
\varepsilon \geq 0 \quad \text { and } \quad \forall i, \quad \varepsilon \geq\left|p_{i}\right|
$$

All the energy conditions mentioned above are local conditions. There exist some other averaged energy conditions that are not of importance to this work. Both local and averaged energy conditions play an essential role in the formulation of classical singularity theorems as the Penrose and the Hawking-Penrose singularity theorems [31], that invokes the weak and strong energy conditions respectively for their proof. Also the proof of the zeroth law of black hole thermodynamics (the constancy of the surface gravity over the event horizon) relies on the dominant energy condition and the proof of the second law of black hole thermodynamics (the area increase theorem) uses the null energy condition [32]. While the classical validity of the energy conditions are perfectly reasonable assumptions, semiclassical quantum effects are capable of violating the null, weak, strong, and dominant energy conditions.

As can be easily seen from (39), the quantum spinor field violates the weak energy condition at the horizon of the black string: the energy density (defined as $\varepsilon=-T_{t}{ }^{t}$ ) is negative on this hypersurface. Further analysis also reveals that at the horizon the dominant energy condition is violated as well. At this point it is interesting to compare the results obtained for the energy conditions in the massive scalar [27, 28] and the massless scalar [26] field cases. The results for the horizon values of the large mass limit of the renormalized stress tensor for massive scalar field on the black static string background reads:

$$
\begin{gathered}
\left.T_{t}{ }^{t}\right|_{\text {horizon }}=-\frac{3}{2} \frac{\alpha^{6} \eta}{\pi^{2} m^{2}}\left(\frac{1}{40}+3 \eta^{2}\right)+\frac{\alpha^{6}}{140 \pi^{2} m^{2}} \\
\left.T_{z}{ }^{z}\right|_{\text {horizon }}=\left.T_{\varphi}{ }^{\varphi}\right|_{\text {horizon }}=-\frac{3}{2} \frac{\alpha^{6} \eta}{\pi^{2} m^{2}}\left(\frac{1}{20}+3 \eta^{2}\right)+\frac{\alpha^{6}}{112 \pi^{2} m^{2}} \\
\left.T_{\rho}{ }^{\rho}\right|_{\text {horizon }}=-\frac{3}{2} \frac{\alpha^{6} \eta}{\pi^{2} m^{2}}\left(\frac{1}{40}+3 \eta^{2}\right)+\frac{\alpha^{6}}{140 \pi^{2} m^{2}}
\end{gathered}
$$


In the general case, all the components of the renormalized stress energy tensor of the quantized massive scalar field will be positive at the horizon for the values of the coupling constant satisfying the relation $3 \eta^{3}+\frac{1}{40} \eta<\frac{1}{210}$. There are some particular cases in which the above relation is always satisfied. The simplest case of the conformal coupling $\xi=\frac{1}{6}$ and the minimal one are two important examples. Also for the case $\xi<\frac{1}{6}$ the components of the quantized scalar field at the horizon are always positive quantities. Then we can conclude that for the particular cases mentioned above the weak energy condition is violated. In the massless scalar field case it is concluded in [26] that the weak, null and strong energy conditions are violated. The precise conditions under which quantum effects in four dimensional spacetime are capable of violating the averaged energy conditions is unknown. The quantum-induced violations of the null, weak, strong, and dominant energy conditions are typically very small. After all, by definition these are order $\hbar$ effects. It is far from clear whether or not it is possible to get a large violation of the energy conditions.

\section{CONCLUDING REMARKS}

The quantization of a massive spinor field coupled to an arbitrary gravitational background space-time was considered. The renormalized quantum mean values of the Lagrangian and the corresponding components of the Energy-Momentum tensor are obtained in the large mass limit using the Schwinger-DeWitt approximation up to the second order in the inverse mass of the field, and are explicitly evaluated in the case of the static black string space-time. The quantum spinor field violates the weak as well as dominant energy conditions at the horizon hypersurface of the black string. This results are expected to be employed in future works to investigate the back-reaction of the quantum spinor field, on the Black String metric.

\section{Acknowledgments}

One of the authors (O.P.F.P) greatly acknowledges M. Chacón Toledo for helpful discussions. We are very much grateful by the helpful remarks of Dr. A. Follaci and Dr. J. Matyjasek, and in addition deeply acknowledge our colleague the MSc. A. Ulacia for a useful introduction to computer algebraic evaluations in General relativity. We also ought 
to express our gratitude by the kind support granted by the Office of External Activities (OEA) of the AS ICTP, under the ICTP Network Net-35.

[1] S. W. Hawking, Commun. Math. Phys 43, 199 (1975) .

[2] N.D. Birrel and P. C. Davies, Quantum Fields in Curved Space, (Cambridge University Press, Cambridge,1982) .

[3] J. P. S. Lemos and V. T. Zanchin, Phys. Rev. D 54, 3840 (1996) .

[4] S. A. Fulling, Aspects of Quantum Field Theory in Curved Space Time, (Cambridge University Press, Cambridge, 1985) .

[5] P. R. Anderson, W. A. Hiscock and D. A. Samuel, Phys. Rev. D 51, 4337 (1995) .

[6] V. P. Frolov and A. I. Zelnikov, Phys. Lett. 115B, 372 (1982), V. P. Frolov and A. I. Zelnikov, Phys. Lett. 123B, 197 (1983), V. P. Frolov and A. I. Zelnikov, Phys. Rev. D 29, 1057 (1984).

[7] A. O. Barvinsky and G. A. Vilkovisky, Phys. Rept. 119, 1 (1985) .

[8] B. S. DeWitt, Phys. Rept 53, 1615 (1984) .

[9] I. G. Avramidi, Nucl. Phys. B 355, 712 (1991), I. G. Avramidi, PhD Thesis, hep-th/9510140.

[10] P. B. Gilkey J. Diff. Geom. 10, 601 (1975) .

[11] J. S. Dowker and R. Critchley, Phys. Rev. D 13, 3224 (1976) .

[12] L. S. Brown and J. P. Cassidy, Phys. Rev. D 15, 2810 (1977) .

[13] T. S. Bunch and P. C. W. Davies, Proc. R. Soc. London A 360, 117 (1978) ;T. S. Bunch J. Phys. A 12, 517 (1979).

[14] B. Allen and A. Folacci, Phys. Rev. D 35, 3771 (1987) ; A. Folacci, J. Math. Phys 32, 2813 (1991) .

[15] K. Kirsten and J. Garriga, Phys. Rev. D 48, 567 (1993) .

[16] K. H. Howard and P. Candelas, Phys. Rev. Lett. 53, 403 (1984) .

[17] P. Candelas, Phys. Rev. D 21, 2185 (1980) .

[18] M. S. Fawcet, Commun. Math. Phys. 89, 103 (1983) .

[19] B. P. Jensen and A. C. Ottewill, Phys. Rev. D 39, 1130 (1989) ; B. P. Jensen, J. G. Mc Laughlin and A. C. Ottewill, Phys. Rev. D 45, 3002 (1992).

[20] P. R. Anderson, W. A. Hiscock, and D. J. Loranz Phys. Rev. Lett. 74, 4365 (1995) .

[21] E. R. Bezerra de Mello, V. B. Bezerra, and N. R. Khusnutdinov Phys. Rev. D 60, 063506 
(1999) .

[22] J. Matyjasek, Phys. Rev. D 61, 124019 (2000) .

[23] J. Matyjasek, Phys. Rev. D 63, 084004 (2001) .

[24] J. Matyjasek, Private Communication .

[25] A. DeBenedictis, Gen. Rel. Grav.31, 1549 (1999).

[26] A. DeBenedictis, Class. Quant. Grav. 16, 1955 (1999).

[27] Owen Pavel Fernández Piedra and Alejandro Cabo Montes de Oca, Phys. Rev. D75, 107501 (2007) .

[28] Owen Pavel Fernández Piedra and Alejandro Cabo Montes de Oca, gr-qc/0701135 .

[29] C. W. Misner, K. S. Thorne and J. A. Wheeler, Gravitation, ( Freeman, San Francisco, 1973).

[30] M. Visser, Lorentzian Wormholes - from Einstein to Hawking, ( American Institute of Physics, New York, 1995).

[31] S. W. Hawking and G. F. R. Ellis, The Large Scale Structure of Space-Time, ( Cambridge University Press, Cambridge, England, 1973).

[32] R. M. Wald, General Relativity, ( Chicago, 1984). 